\title{
Broadband Metallic Fiber-to-Chip Couplers and a Low-Complexity Integrated Plasmonic Platform
}

\section{Journal Article}

\section{Author(s):}

Messner, Andreas (D); Jud, Pascal A.; Winiger, Joel; Eppenberger, Marco (D); Chelladurai, Daniel (1); Heni, Wolfgang (i); Bäuerle, Benedikt (1D; Koch, Ueli (D); Ma, Ping (1); Haffner, Christian (1); Xu, Huajun; Elder, Delwin L.; Dalton, Larry R.; Smajic, Jasmin (1D; Leuthold, Juerg (1)

\section{Publication date:}

2021-06-09

\section{Permanent link:}

https://doi.org/10.3929/ethz-b-000493455

\section{Rights / license:}

Creative Commons Attribution-NonCommercial-NoDerivatives 4.0 International

\section{Originally published in:}

Nano Letters 21(11), https://doi.org/10.1021/acs.nanolett.0c05069

\section{Funding acknowledgement:}

670478 - Plasmonic-Silicon-Organic Hybrid - a Universal Platform for THz Communications (EC)

780997 - Wafer-scale, CMOS integration of photonics, plasmonics and electronics for mass manufacturing $200 \mathrm{~Gb} / \mathrm{s} \mathrm{NRZ}$ transceivers towards low-cost Terabit connectivity in Data Centers (EC)

871391 - Energy- and Size-efficient Ultra-fast Plasmonic Circuits for Neuromorphic Computing Architectures (EC) 


\section{Broadband Metallic Fiber-to-Chip Couplers and a Low-Complexity Integrated Plasmonic Platform}

Andreas Messner, * Pascal A. Jud, Joel Winiger, Marco Eppenberger, Daniel Chelladurai, Wolfgang Heni, Benedikt Baeuerle, Ueli Koch, Ping Ma, Christian Haffner, Huajun Xu, Delwin L. Elder, Larry R. Dalton, Jasmin Smajic, and Juerg Leuthold*

Cite This: Nano Lett. 2021, 21, 4539-4545

ABSTRACT: We present a plasmonic platform featuring efficient, broadband metallic fiber-to-chip couplers that directly interface plasmonic slot waveguides, such as compact and high-speed electro-optic modulators. The metallic gratings exhibit an experimental fiber-to-slot coupling efficiency of $-2.7 \mathrm{~dB}$ with $-1.4 \mathrm{~dB}$ in simulations with the same coupling principle. Further, they offer a huge spectral window with a $3 \mathrm{~dB}$ passband of $350 \mathrm{~nm}$. The technology relies on a vertically arranged layer stack, metalinsulator-metal waveguides, and fiber-to-slot couplers and is formed in only one lithography step with a minimum feature size of $250 \mathrm{~nm}$. As an application example, we fabricate new modulator

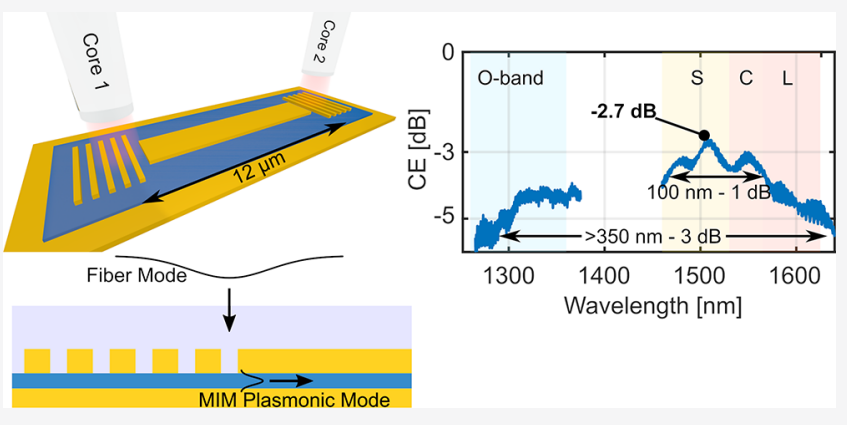
devices with an electro-optic organic material in the slot waveguide and reach 50 and $100 \mathrm{Gbit} / \mathrm{s}$ data modulation in the O- and C-bands within the same device. The devices' broad spectral bandwidth and their relaxed fabrication may render them suitable for experiments and applications in the scope of sensing, nonlinear optics, or telecommunications.

KEYWORDS: Plasmonics, slot waveguides, grating, fiber-to-chip coupling, extraordinary optical transmission, electro-optic modulator

$\mathrm{O}$ n-chip integrated functional plasmonic waveguides offer a broad variety of functionality in science and engineering. The high subwavelength confinement of electromagnetic waves at metal-insulator interfaces gives rise to a strong light-matter interaction and allows for applications such as the observation of nonlinear phenomena, ${ }^{1,2}$ sensing, ${ }^{3}$ or modulation and detection in optical communications. ${ }^{4-10}$ Two building blocks are indispensable for such on-chip devices: First, an active plasmonic waveguide and, second, a coupler to connect it with the photonic world.

A variety of plasmonic waveguides has been described in the literature. $^{11}$ Plasmonic metal-insulator-metal (MIM) slot waveguides $^{12-15}$ stand out as they provide extreme field confinement and their metals can simultaneously act as highspeed electrical contacts. ${ }^{16}$ These MIM slot waveguides are usually formed by a horizontal, in-plane gap in a single metal layer and have been extensively used in experiments and fibercoupled applications. ${ }^{6,9,17-19}$ Until now, however, experimental realizations of MIM waveguides formed by a vertical, out-ofplane gap between two metal layers have often been with passive devices and rarely fiber or waveguide coupled. ${ }^{20-27}$ The reasons for this might be due to both the difficulties of optical coupling and the functionalization with active materials.

On the other hand, very efficient photonic-to-plasmonic couplers have been conceived to couple plasmonic MIM slots to photonic bus waveguides ${ }^{17,28}$ or to guided optical fiber modes by either photonic grating couplers ${ }^{19,29}$ or metallic structures. ${ }^{4,30}$ These achievements are particularly remarkable, because plasmonic MIM modes are confined to areas below the diffraction limit, typically $\sim(100 \times 100) \mathrm{nm}^{2}$, and have a very high momentum, whereas photonic modes are considerably larger with a lower momentum. Both facts make it difficult to couple to a $\mu \mathrm{m}$-sized, dielectrically guided photonic mode.

This Letter presents a new fiber-to-plasmonic waveguide interface, which is not only efficient, broadband, and simple to fabricate but also allows one to tap the advantages of a vertically stacked plasmonic platform (VSPP). Coupling efficiencies as low as -1.4 and $-2.7 \mathrm{~dB}$ with a $350 \mathrm{~nm}$-wide $3 \mathrm{~dB}$ spectral window are predicted in a simulation and demonstrated in an experiment, respectively. Besides the passive demonstration of the coupler, the active proof-of-principle devices relying on the

Received: December 24, 2020

Revised: April 22, 2021

Published: May 18, 2021 
same geometry are fabricated and tested for operation up to 100 Gbit/s.
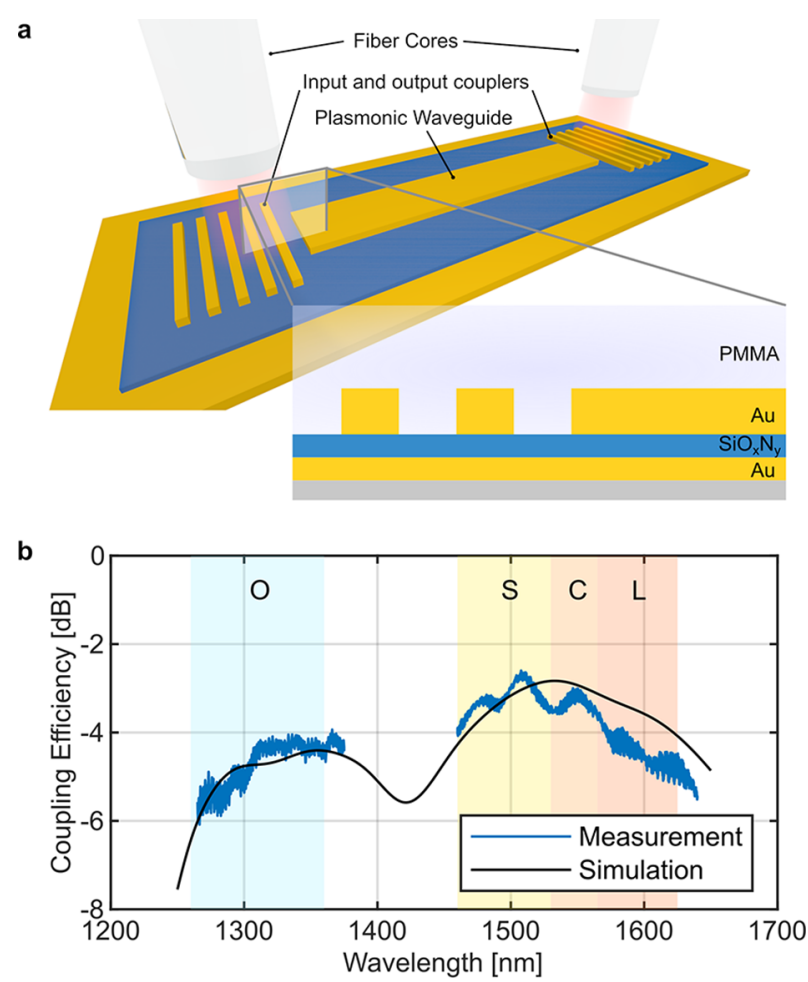

Figure 1. Vertically stacked plasmonic platform (VSPP). (a) Schematic of a plasmonic MIM waveguide with two fiber-to-slot couplers. The inset shows a cross-sectional view of the coupler and the MIM waveguide. (b) Simulated and measured fiber-to-slot coupling efficiency in the O-, S-, C-, and L-bands.

Figure 1a shows a schematic of the demonstrated platform. Its MIM slot waveguide and the fiber-to-slot couplers are formed by a stack of a bottom metal, an insulator, and a structured top metal layer; see the cross-section in the figure inset, similarly as
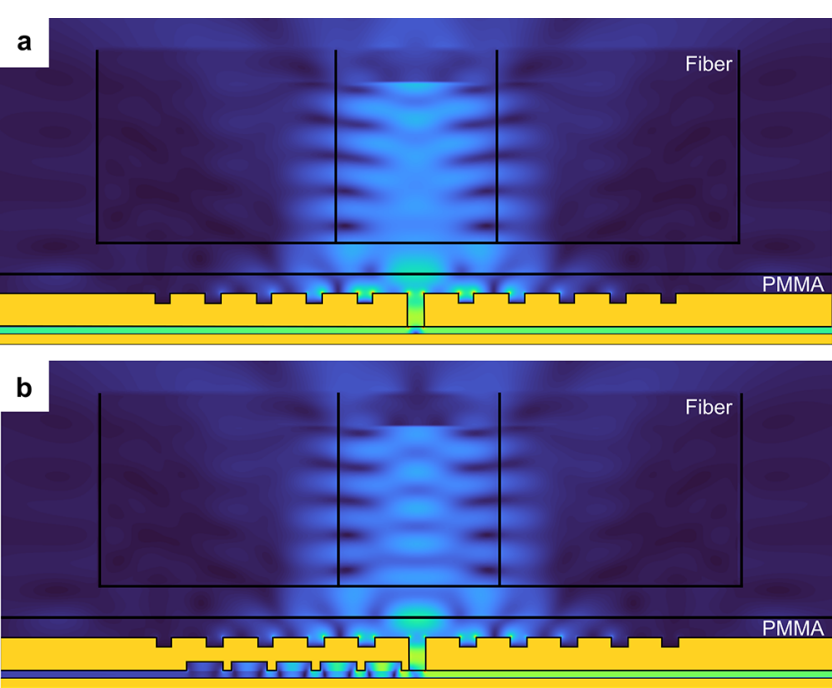

Figure 3. Simulated absolute value of the electric field of two structures with only one aperture and corrugations. The fiber is centered above the aperture. (a) Periodic corrugations only in the upper surface of the top metal. As no feature can provide directionality, the power coupled to the structure is fed into the left and right plasmonic MIM waveguide in equal parts. (b) The same structure with an additional grating on the bottom surface of the left metal. This grating serves as a reflector for the MIM mode, and the coupled power is unidirectionally fed into the right waveguide.

introduced for coupling from a microscope objective to a vertical MIM waveguide. ${ }^{25}$ The fiber mode from a pitch-reduced optical fiber array (Chiral Photonics) impinges on one of the grating sections, which directly maps the signal into the plasmonic waveguide. After plasmonic propagation, a second, identical metal grating couples the light back to a second fiber core. The device layout is described in Supplementary Section 1.

The device is fabricated layer by layer, as detailed in Supplementary Section 2. The top metal is structured in one single lithography step. An alignment process with tolerances in the $\mu \mathrm{m}$ range is only required if electrical contacts need to be structured; otherwise, no alignment is needed at all.
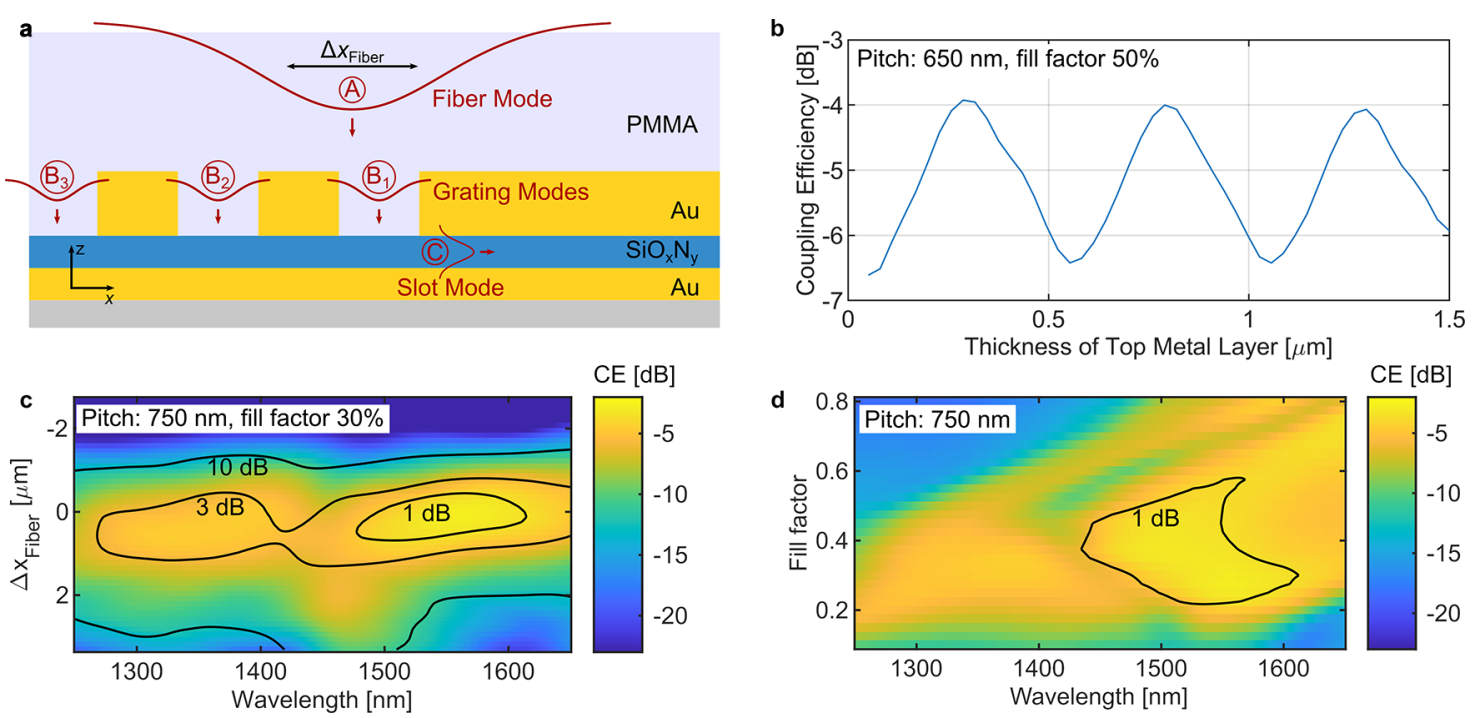

Figure 2. (a) Coupling mechanism: The fiber mode $A$ couples to intermediate "grating modes" $B_{n}$, which then launch the MIM slot mode $C$. (b) The coupling efficiency's periodic dependence on the top metal thickness. (c) Sensitivity of the coupling efficiency to the lateral position of the fiber and (d) to a change of fill factor. 

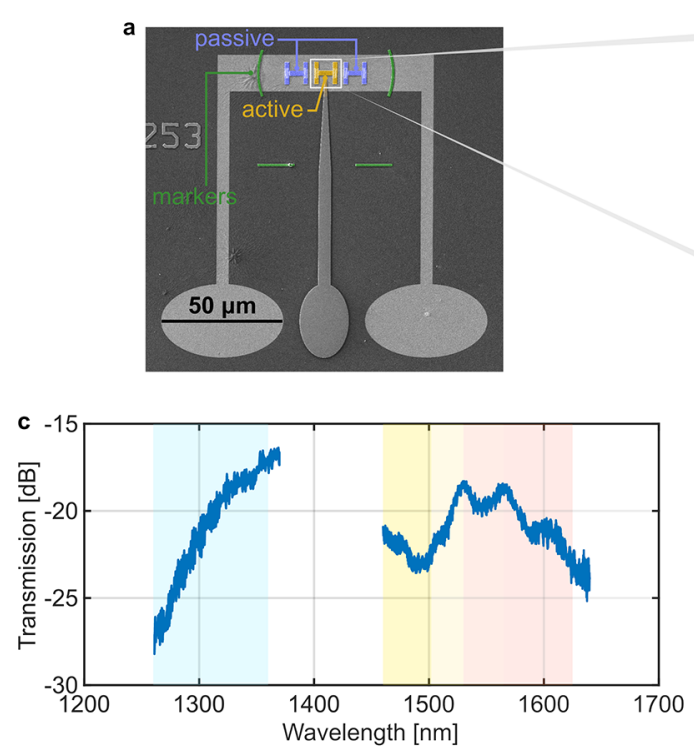

e

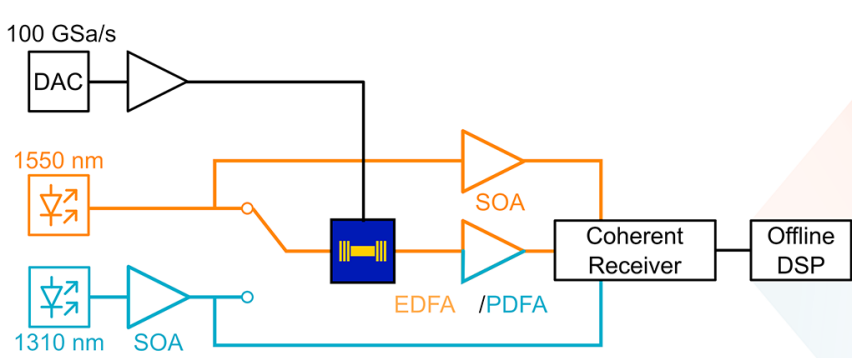

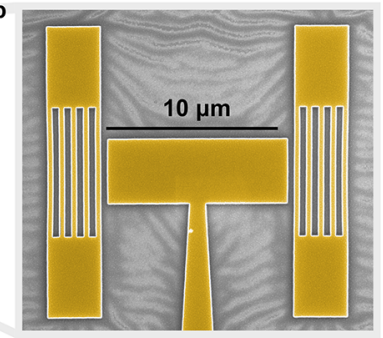
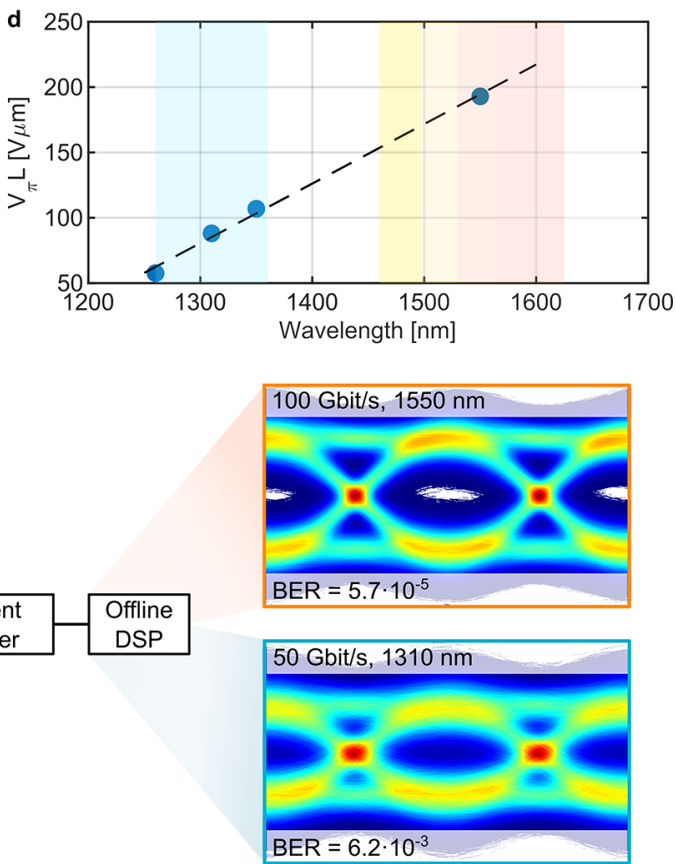

Figure 4. (a) SEM image of an active device (upper metal layer: yellow) engulfed by two passive ones (blue). Markers for optical fiber positioning shown in green. (b) Close-up of the active device with narrow lateral contact. (c) Fiber-to-fiber transmission of the active device. Peak fiber-to-fiber transmission is $-16.7 \mathrm{~dB}$ at $1370 \mathrm{~nm}$. (d) Half-wave voltage-length product $\left(V_{\pi} L\right)$ measurements, representing the inverse modulation efficiency, in one device at different wavelengths. At shorter wavelengths, a lower drive voltage is required to shift the light's phase by $\pi$. (e) Employed data modulation setup. The insets show the eye diagrams of a $100 \mathrm{Gbit} / \mathrm{s}$ signal at $1550 \mathrm{~nm}$ and a $50 \mathrm{Gbit} / \mathrm{s}$ signal at $1310 \mathrm{~nm}$.

In order to verify the capabilities of the VSPP, we experimentally demonstrate a passive structure featuring fiberto-slot converters with an optical bandwidth ranging from 1300 to $1600 \mathrm{~nm}$ and coupling efficiencies of $-2.7 \mathrm{~dB}$; see Figure $1 \mathrm{~b}$. Furthermore, we demonstrate the functionalization of the VSPP by fabricating a broadband and high-speed electro-optic modulator that supports both $\mathrm{O}$ - and C-band operation and data modulation up to $100 \mathrm{Gbit} / \mathrm{s}$. This comes at the cost of a reduced coupling efficiency due to an early stage fabrication process (see Supplementary Section 2), and the coupling penalty is not of a fundamental nature.

The VSPP with MIM waveguides and fiber-to-slot coupler have several distinct advantages, some of which have already been discussed in the works of Gramotnev et al. ${ }^{31}$ and Nielsen and Bozhevolnyi. ${ }^{25}$ First, the cross-section of the plasmonic modulator is well-defined, as the thickness of the individual layers can be controlled precisely during deposition. ${ }^{31}$ The layered approach allows one to achieve arbitrarily large slot aspect ratios, $^{31}$ while conventional horizontal MIM waveguides are typically limited to aspect ratios below $\sim 5: 1$ due to lithographic constraints. In consequence, the plasmonic mode is very strongly confined within the slot. This helps to reduce scattering into leaky and unwanted edge modes ${ }^{23}$ and provides an excellent field overlap with an RF field, in case the waveguide is functionalized with electro-optic materials, either organic ${ }^{32}$ via spin-coating or ferroelectric such as $\mathrm{BaTiO}_{3}{ }^{33}$ or $\mathrm{LiNbO}_{3}{ }^{10}$ via metal-to-metal direct wafer bonding. ${ }^{34}$ Furthermore, leakage into the substrate, which can be observed for plasmonic MIM slot waveguides with a low aspect ratio and low-index material in the slot $^{33}$ is completely suppressed here. This offers a way to implement plasmonic modulators on high-index substrates or plasmonic modulators with a large gap size. The latter might be beneficial for the modulation efficiency of devices based on both organic electro-optic or ferroelectric materials to reduce the influence of boundary effects at the metal-insulator interfaces. ${ }^{36}$ Second, instead of relying on plasmonic slot waveguides that were fabricated with an etching or a lift-off process, the metal surfaces defining the waveguide can be ultrasmooth. ${ }^{37}$ This lowers plasmonic propagation losses, ${ }^{38,39}$ particularly in the case of small gap size. ${ }^{40}$ Third, the fabrication process of this new type of high-speed plasmonic modulator is considerably simpler than that of the established, horizontal modulators. ${ }^{25,31}$ All features, even those of the metallic grating couplers, are larger than $250 \mathrm{~nm}$. This alleviates lithography requirements as compared to the horizontal MIM structures where sub-100 nm features must be structured and aligned with the help of advanced lithography technologies, such as e-beam. Fourth, despite their simplicity in structure and fabrication, the fiber-to- 
slot coupling has low loss and extraordinary broadband. Figure $1 \mathrm{~b}$ shows the wavelength-dependent fiber-to-slot coupling efficiency of $-2.7 \pm 0.5 \mathrm{~dB}$ at $1510 \mathrm{~nm}$ with a $3 \mathrm{~dB}$ bandwidth of $350 \mathrm{~nm}$, spreading over the O-, S-, C-, and L-bands. The $1 \mathrm{~dB}$ bandwidth of $\sim 100 \mathrm{~nm}$ is comparable to that reported for other metallic gratings. ${ }^{41}$ The plasmonic losses of $\alpha=0.5 \pm 0.1 \mathrm{~dB} / \mu \mathrm{m}$ at $\lambda=1550 \mathrm{~nm}$ have been extracted from cut-back measurements of 11,23 , and $35 \mu$ m-long devices with a gap size of 100 $\mathrm{nm}$. The band between 1375 and $1460 \mathrm{~nm}$ has not been accessible with our measurement setup. The experimental behavior is in very good agreement with $2 \mathrm{D}$ finite-difference time-domain (FDTD) simulations, ${ }^{42}$ depicted as the dark line. These simulations have been carried out with the device's geometrical properties and the grating period adapted from 800 to $750 \mathrm{~nm}$. This adaptation is attributed to imperfect fabrication in terms of the dimensions and material refractive indices. More detailed information on the simulations can be found in Supplementary Section 3. The dip of the simulated transmission spectrum at $\sim 1430 \mathrm{~nm}$ can be attributed to a Bragg passband of the grating; see Supplementary Section 4.

The fiber-to-slot coupling mechanism is depicted in Figure 2a and can be separated in two independent parts: First, the fiber mode $A$ with a $2 \mu \mathrm{m}$ mode field diameter is coupled to plasmonic MIM modes $B_{n}$ that are guided in between the grating bars and travel downward. Second, these modes are coupled to the actual slot waveguide mode $C$, where the grating acts as a Bragg reflector; see Supplementary Section 4. The independence of these coupling mechanisms is revealed by a sweep of the upper metal layer's thickness, best seen in a model with a period of 650 $\mathrm{nm}$ and a fill factor of $50 \%$. This is in contrast to a classical grating coupling scheme, ${ }^{43}$ where the grating provides the missing momentum to the incoming light in order to match with the momentum of the MIM mode. ${ }^{44}$ Figure $2 \mathrm{~b}$ shows the results, where optima of the coupling efficiency occur every $490 \mathrm{~nm}$ of thickness, which corresponds to exactly half the wavelength of a MIM slot plasmon polariton traveling in a $325 \mathrm{~nm}$-wide gold slot filled with PMMA. This indicates that the grating itself acts as a Fabry-Pérot (FP) resonator. ${ }^{45-47}$ In this, the presented coupler is closely related to the phenomenon of extraordinary optical transmission (EOT), ${ }^{46-49}$ where plasmonic modes enable more light to be transmitted through an aperture in a metal sheet than would be expected from the aperture size alone. Typically, in EOT, a grating or periodic array of features serves to match the momentum of the incident, free-space wave to that of surface plasmons. Another effect that can strongly contribute to EOT is mediated by FP cavity modes in the grating structure itself. $^{45-47,50}$ The importance of these FP modes in the EOT through one-dimensional metal gratings has first been recognized by Porto et al. ${ }^{45}$ and has subsequently been the subject of several studies. ${ }^{45,51-53}$ In the structure and wavelength regime of this work, we have strong evidence that we operate in the FP-dominated regime: First, we see the strong dependence of the coupling efficiency at a certain wavelength on the gold thickness and, hence, on the FP resonance condition. Second, we operate at rather long wavelengths, where FP modes dominate, ${ }^{45,51,52,54}$ and third, we operate at normal incidence, where again FP plays a much more important role than coupled plasmon resonances. $^{54}$

For practical reasons, it is also important to investigate the coupler's dependence on geometrical parameters. Figure $2 c$ shows the coupling efficiency for a sweep of the lateral fiber position. The coupling efficiency stays within a $1 \mathrm{~dB}$ range for a displacement $\Delta x_{\text {Fiber }}$ of less than $\pm 0.5 \mu \mathrm{m}$ from the optimal position. The fill factor, i.e., the fraction of the period fill with metal, can be subject to fabrication derivations, whereas the grating period itself is usually well controlled in fabrication. Figure $2 \mathrm{~d}$ shows the influence of a change in fill factor on the coupling efficiency: The penalty is below $1 \mathrm{~dB}$ for a fill factor of $30 \pm 10 \%$, which corresponds to a rather relaxed required dimension accuracy of $150 \mathrm{~nm}$. A more in-depth study on the influence of fiber mode size, the number of grating bars, and the width of the waveguide and gratings is included in Supplementary Section 5.

As has been shown above, the grating modes $B_{n}$ play a major role in the fiber-to-slot coupling mechanism. The fabricated grating has five gaps, and it is interesting to find out their contribution to the overall coupling. To this end, MIM waveguide mode $C$ has been excited in simulation, and the power transmitted to the modes $B_{1}, B_{2}$, and $B_{3}$ has been determined. The coupling coefficients from the excited port to the grating modes $B_{1}, B_{2}$, and $B_{3}$ are $S_{\mathrm{CB} 1}=-2.0 \mathrm{~dB}, S_{\mathrm{CB} 2}=-9.9$ $\mathrm{dB}$, and $S_{\mathrm{CB} 3}=-17.8 \mathrm{~dB}$. We can conclude that the first grating aperture is the most important for the total coupling efficiency.

To investigate the structure and the coupling effect further, we conceive a coupling structure with only one aperture, such as shown in Figure 3a. The aperture is engulfed by a shallow grating on the cladding side, which helps to collect the incident light and guide it to the grating opening. ${ }^{1,49,55}$ The top metal thickness was set to $535 \mathrm{~nm}$, as is possible without penalty; see Figure $2 \mathrm{~b}$. A particle swarm optimizer has been used to find the shallow grating's pitch of $793 \mathrm{~nm}$, fill factor of 69\%, and depth of $150 \mathrm{~nm}$. Although the structure lacks elements that could provide directionality, the CE into one MIM waveguide mode is $S_{\mathrm{CA}}=$ $-4.7 \mathrm{~dB}$. In a second structure (see Figure $3 \mathrm{~b}$ ), an additional, shallow grating has been introduced on the substrate side (pitch: $694 \mathrm{~nm}$; fill factor: 20\%; depth: $135 \mathrm{~nm}$ ). This grating acts as a reflector for the MIM waveguide mode and provides directionality to the structure, so that the simulated coupling efficiency rises to $S_{\mathrm{CA}}=-1.4 \mathrm{~dB}$. While the fabrication of these independent, shallow gratings requires more effort than that of the fully etched couplers, the result highlights the close relation of the presented couplers with the principles of EOT and shows a potential way to optimize these structures. Another way to optimize the structure without adding either too much fabricational complexity or more lithographic steps is to follow the ideas of Søndergaard et al. and tilt the sidewall angles of the grating bars. ${ }^{56}$ Preliminary simulations show that the CE can be improved by $\sim 0.5 \mathrm{~dB}$.

In a final demonstration, we return to the idea of the fully etched couplers and fabricate a vertically stacked plasmonic phase modulator as an active application example. ${ }^{57}$ Electron micrographs are shown in Figure 4a,b. Instead of the $100 \mathrm{~nm}$ thick SiON layer of the device described above, a $10 \mathrm{~nm}$-thick $\mathrm{TiO}_{2}$ charge-blocking layer ${ }^{58}$ and a $140 \mathrm{~nm}$-thick film of the organic electro-optic material HLD $1 / 2^{59}$ have been sandwiched between the top and bottom metal electrodes. The fiber-to-fiber transmission peak is $-16.7 \mathrm{~dB}$ at $1370 \mathrm{~nm}$ (see Figure 4c) and is decreased as compared to the passive device. This is due to fabrication challenges when structuring an upper metal sheet on top of an organic layer.

Electro-optic characterization was carried out as described in Supplementary Section 6. A sinusoidal radio frequency signal was fed to the chip, and the modulation efficiency was measured at several wavelength points in the same device. Figure $4 \mathrm{~d}$ shows that the modulation is stronger at lower wavelengths, closer to the resonance of the OEO molecules. This behavior reproduces 
the findings of Haffner et al. with a similar chromophore in horizontal MIM slot waveguide modulators ${ }^{60}$ and serves as strong evidence that the light is coupled through the actual vertically stacked slot waveguide at all wavelengths and is not transmitted as a long-range surface plasmon polariton on top of the waveguide.

Eventually, the data modulation capacity of the phase shifter was tested in the $\mathrm{O}$ - and $\mathrm{C}$-bands. Figure $4 \mathrm{e}$ shows the schematic of the data modulation setup and the received eye diagrams; see Supplementary Section 7 for details. A bit rate of $100 \mathrm{Gbit} / \mathrm{s}$ could be modulated and received at $1550 \mathrm{~nm}$ with a bit error ratio (BER) of $5.7 \times 10^{-5}$. At $1310 \mathrm{~nm}, 50 \mathrm{Gbit} / \mathrm{s}$ could be modulated and received with a BER of $6.2 \times 10^{-3}$. The performance penalty in the O-band might seem surprising, as both the insertion loss and the modulation efficiency are superior in the O-band. However, the available coherent receiver has been designed for C-band operation and was operated outof-specifications to receive the O-band signal.

The presented vertical plasmonic platform offers a way to employ out-of-plane, vertically stacked plasmonic MIM slot waveguides. It features efficient and ultrabroadband coupling from a photonic fiber mode to the plasmonic MIM waveguide (350 nm-wide spectral passband with a peak coupling efficiency of $-2.7 \mathrm{~dB}$ ) and a simple fabrication, requiring neither precise alignment nor features smaller than $250 \mathrm{~nm}$. This fact sets it apart from conventional plasmonic MIM waveguides that are dependent on e-beam lithography. In an application example, we employ the new platform and fabricate a plasmonic-organic hybrid electro-optic modulator. While the measured data rate of $100 \mathrm{Gbit} / \mathrm{s}$ is state-of-the art, the device benefits from the simple fabrication and can be operated both in the C-and O-bands. We think that the presented platform lowers the complexity of plasmonic devices and makes experiments in the plasmonic regime more easily achievable. This would benefit applications in science, technology, and engineering.

\section{ASSOCIATED CONTENT}

\section{(s) Supporting Information}

The Supporting Information is available free of charge at https://pubs.acs.org/doi/10.1021/acs.nanolett.0c05069.

Device design and fabrication, the fiber-to-slot coupling mechanism and the influence of the device dimensions on the coupling efficiency, and details of the optical and electro-optical characterization experiments (PDF)

\section{AUTHOR INFORMATION}

\section{Corresponding Authors}

Andreas Messner - ETH Zurich, Institute of Electromagnetic Fields (IEF), 8092 Zürich, Switzerland; ○ orcid.org/00000002-7849-9644; Email: amessner@ethz.ch

Juerg Leuthold - Polariton Technologies AG, 8803 Rüschlikon, Switzerland; ETH Zurich, Institute of Electromagnetic Fields (IEF), 8092 Zürich, Switzerland; (1) orcid.org/0000-00030111-8169; Email: leuthold@ethz.ch

\section{Authors}

Pascal A. Jud - ETH Zurich, Institute of Electromagnetic Fields (IEF), 8092 Zürich, Switzerland; Present Address: P.A.J.: DECTRIS Ltd., Taefernweg 1, 5405 Baden-Daettwil, Switzerland.

Joel Winiger - ETH Zurich, Institute of Electromagnetic Fields (IEF), 8092 Zürich, Switzerland
Marco Eppenberger - ETH Zurich, Institute of Electromagnetic Fields (IEF), 8092 Zürich, Switzerland

Daniel Chelladurai - ETH Zurich, Institute of Electromagnetic Fields (IEF), 8092 Zürich, Switzerland

Wolfgang Heni - Polariton Technologies AG, 8803 Rüschlikon, Switzerland

Benedikt Baeuerle - Polariton Technologies AG, 8803 Rüschlikon, Switzerland

Ueli Koch - ETH Zurich, Institute of Electromagnetic Fields (IEF), 8092 Zürich, Switzerland

Ping Ma - ETH Zurich, Institute of Electromagnetic Fields (IEF), 8092 Zürich, Switzerland; ㅇ orcid.org/0000-00030674-8530

Christian Haffner - ETH Zurich, Institute of Electromagnetic Fields (IEF), 8092 Zürich, Switzerland; Present Address: C.H.: IMEC, 3001 Leuven, Belgium.

Huajun Xu - University of Washington, Department of Chemistry, Seattle, Washington 98195-1700, United States; (1) orcid.org/0000-0002-5267-5910

Delwin L. Elder - University of Washington, Department of Chemistry, Seattle, Washington 98195-1700, United States; (1) orcid.org/0000-0001-9302-3858

Larry R. Dalton - University of Washington, Department of Chemistry, Seattle, Washington 98195-1700, United States

Jasmin Smajic - ETH Zurich, Institute of Electromagnetic Fields (IEF), 8092 Zürich, Switzerland

Complete contact information is available at:

https://pubs.acs.org/10.1021/acs.nanolett.0c05069

\section{Author Contributions}

A.M., P.A.J., J.W., P.M., C.H., and J.L. have developed the device and coupling concept. A.M., P.A.J., J.W., U.K., C.H., and P.M. have designed the devices, which have been fabricated by A.M., P.A.J., and P.M. H.X., D.L.E., and L.R.D. have synthesized the organic electro-optic material HLD1/HLD2, which has been processed and deposited by W.H. A.M., P.A.J., and M.E. have carried out the measurements with the support of W.H. and B.B. for data measurements. A.M., P.A.J., U.K., D.C., and J.S. have carried out the simulations. All authors have contributed to the writing of the manuscript.

\section{Notes}

The authors declare the following competing financial interest(s): W.H., B.B., and J.L. are involved in activities to commercialize plasmonic modulators at Polariton Technologies Ltd. The remaining authors declare no competing interests.

\section{ACKNOWLEDGMENTS}

This work was carried out in the Binning and Rohrer Nanotechnology Center. The authors acknowledge financial support from the ERC under grants 670478 PLASILOR, H2020-780997 plaCMOS, H2020-863155 NEBULA, and H2020-871391 PlasmoniAC and from the ASFOR, Grant FA9550-19-1-0069. We thank the Cleanroom Operations Team of the Binnig and Rohrer Nanotechnology Center (BRNC) for their help and support. A.M. thanks Henri Lezec for the fruitful discussion on the functional principle of the coupling mechanism.

\section{ABBREVIATIONS}

MIM, metal-insulator-metal; VSPP, vertically stacked plasmonic platform; BER, bit error ratio; CE, Coupling Efficiency; EOT, Extraordinary Optical Transmission; FP, Fabry-Pérot 


\section{REFERENCES}

(1) Cai, W.; Vasudev, A. P.; Brongersma, M. L. Electrically Controlled Nonlinear Generation of Light with Plasmonics. Science 2011, 333 (6050), 1720-1723.

(2) Davidson, R. B.; Yanchenko, A.; Ziegler, J. I.; Avanesyan, S. M.; Lawrie, B. J.; Haglund, R. F. Ultrafast Plasmonic Control of Second Harmonic Generation. ACS Photonics 2016, 3 (8), 1477-1481.

(3) Stockman, M. I.; Kneipp, K.; Bozhevolnyi, S. I.; Saha, S.; Dutta, A.; Ndukaife, J.; Kinsey, N.; Reddy, H.; Guler, U.; Shalaev, V. M.; Boltasseva, A.; Gholipour, B.; Krishnamoorthy, H. N. S.; MacDonald, K. F.; Soci, C.; Zheludev, N. I.; Savinov, V.; Singh, R.; Groß, P.; Lienau, C.; Vadai, M.; Solomon, M. L.; Barton, D. R.; Lawrence, M.; Dionne, J. A.; Boriskina, S. V.; Esteban, R.; Aizpurua, J.; Zhang, X.; Yang, S.; Wang, D.; Wang, W.; Odom, T. W.; Accanto, N.; de Roque, P. M.; Hancu, I. M.; Piatkowski, L.; van Hulst, N. F.; Kling, M. F. Roadmap on plasmonics. J. Opt. 2018, 20 (4), 043001.

(4) Ayata, M.; Fedoryshyn, Y.; Heni, W.; Baeuerle, B.; Josten, A.; Zahner, M.; Koch, U.; Salamin, Y.; Hoessbacher, C.; Haffner, C.; Elder, D. L.; Dalton, L. R.; Leuthold, J. High-speed plasmonic modulator in a single metal layer. Science 2017, 358 (6363), 630-632.

(5) Heni, W.; Fedoryshyn, Y.; Baeuerle, B.; Josten, A.; Hoessbacher, C. B.; Messner, A.; Haffner, C.; Watanabe, T.; Salamin, Y.; Koch, U.; Elder, D. L.; Dalton, L. R.; Leuthold, J. Plasmonic IQ modulators with attojoule per bit electrical energy consumption. Nat. Commun. 2019, 10 (1), 1694.

(6) Haffner, C.; Chelladurai, D.; Fedoryshyn, Y.; Josten, A.; Baeuerle, B.; Heni, W.; Watanabe, T.; Cui, T.; Cheng, B.; Saha, S.; Elder, D. L.; Dalton, L. R.; Boltasseva, A.; Shalaev, V. M.; Kinsey, N.; Leuthold, J. Low-loss plasmon-assisted electro-optic modulator. Nature 2018, 556 (7702), 483-486.

(7) Heni, W.; Haffner, C.; Baeuerle, B.; Fedoryshyn, Y.; Josten, A.; Hillerkuss, D.; Niegemann, J.; Melikyan, A.; Kohl, M.; Elder, D. L.; Dalton, L. R.; Hafner, C.; Leuthold, J. 108 Gbit/s Plasmonic MachZehnder Modulator with $>70 \mathrm{GHz}$ Electrical Bandwidth. J. Lightwave Technol. 2016, 34 (2), 393-400.

(8) Ma, P.; Salamin, Y.; Baeuerle, B.; Josten, A.; Heni, W.; Emboras, A.; Leuthold, J. Plasmonically Enhanced Graphene Photodetector Featuring 100 Gbit/s Data Reception, High Responsivity, and Compact Size. ACS Photonics 2019, 6 (1), 154-161.

(9) Salamin, Y.; Ma, P.; Baeuerle, B.; Emboras, A.; Fedoryshyn, Y.; Heni, W.; Cheng, B.; Josten, A.; Leuthold, J. 100 GHz Plasmonic Photodetector. ACS Photonics 2018, 5 (8), 3291-3297.

(10) Thomaschewski, M.; Zenin, V. A.; Wolff, C.; Bozhevolnyi, S. I. Plasmonic monolithic lithium niobate directional coupler switches. Nat. Commun. 2020, 11 (1), 748.

(11) Gramotnev, D. K.; Bozhevolnyi, S. I. Plasmonics beyond the diffraction limit. Nat. Photonics 2010, 4 (2), 83-91.

(12) Tanaka, K.; Tanaka, M. Simulations of nanometric optical circuits based on surface plasmon polariton gap waveguide. Appl. Phys. Lett. 2003, 82 (8), 1158-1160.

(13) Veronis, G.; Fan, S. Guided subwavelength plasmonic mode supported by a slot in a thin metal film. Opt. Lett. 2005, 30 (24), 33593361 .

(14) Liu, L.; Han, Z.; He, S. Novel surface plasmon waveguide for high integration. Opt. Express 2005, 13 (17), 6645-6650.

(15) Pile, D. F. P.; Ogawa, T.; Gramotnev, D. K.; Matsuzaki, Y.; Vernon, K. C.; Yamaguchi, K.; Okamoto, T.; Haraguchi, M.; Fukui, M. Two-dimensionally localized modes of a nanoscale gap plasmon waveguide. Appl. Phys. Lett. 2005, 87 (26), 261114-261114.

(16) Burla, M.; Hoessbacher, C.; Heni, W.; Haffner, C.; Fedoryshyn, Y.; Werner, D.; Watanabe, T.; Massler, H.; Elder, D. L.; Dalton, L. R.; Leuthold, J. $500 \mathrm{GHz}$ plasmonic Mach-Zehnder modulator enabling sub-THz microwave photonics. APL Photonics 2019, 4 (5), 056106.

(17) Melikyan, A.; Alloatti, L.; Muslija, A.; Hillerkuss, D.; Schindler, P. C.; Li, J.; Palmer, R.; Korn, D.; Muehlbrandt, S.; Van Thourhout, D.; Chen, B.; Dinu, R.; Sommer, M.; Koos, C.; Kohl, M.; Freude, W.; Leuthold, J. High-speed plasmonic phase modulators. Nat. Photonics 2014, 8 (3), 229-233.
(18) Nielsen, M. P.; Shi, X.; Dichtl, P.; Maier, S. A.; Oulton, R. F. Giant nonlinear response at a plasmonic nanofocus drives efficient fourwave mixing. Science 2017, 358 (6367), 1179.

(19) Nielsen, M. P.; Lafone, L.; Rakovich, A.; Sidiropoulos, T. P. H.; Rahmani, M.; Maier, S. A.; Oulton, R. F. Adiabatic Nanofocusing in Hybrid Gap Plasmon Waveguides on the Silicon-on-Insulator Platform. Nano Lett. 2016, 16 (2), 1410-1414.

(20) Dionne, J. A.; Diest, K.; Sweatlock, L. A.; Atwater, H. A. PlasMOStor: a Metal-Oxide-Si Field Effect Plasmonic Modulator. Nano Lett. 2009, 9 (2), 897-897.

(21) Yang, R.; Lu, Z. Silicon-on-Insulator Platform for Integration of 3-D Nanoplasmonic Devices. IEEE Photonics Technol. Lett. 2011, 23 (22), 1652-1654.

(22) Choo, H.; Kim, M.-K.; Staffaroni, M.; Seok, T. J.; Bokor, J.; Cabrini, S.; Schuck, P. J.; Wu, M. C.; Yablonovitch, E. Nanofocusing in a metal-insulator-metal gap plasmon waveguide with a three-dimensional linear taper. Nat. Photonics 2012, 6 (12), 838-844.

(23) Chen, Y.; Wang, J.; Chen, X.; Yan, M.; Qiu, M. Plasmonic analog of microstrip transmission line and effect of thermal annealing on its propagation loss. Opt. Express 2013, 21 (2), 1639-1644.

(24) Dennis, B. S.; Haftel, M. I.; Czaplewski, D. A.; Lopez, D.; Blumberg, G.; Aksyuk, V. A. Compact nanomechanical plasmonic phase modulators. Nat. Photonics 2015, 9 (4), 267-273.

(25) Nielsen, M. G.; Bozhevolnyi, S. I. Highly confined gap surface plasmon modes in metal strip-gap-film configurations. J. Opt. Soc. Am. B 2015, 32 (3), 462-467.

(26) Messner, A.; Jud, P. A.; Heni, W.; Koch, U.; Xu, H.; Elder, D. L.; Dalton, L. R.; Ma, P.; Leuthold, J. Vertically Stacked Plasmonic Slot Waveguide with $100 \mathrm{Gbit} / \mathrm{s}$ Electro-Optical Modulation. In 9th International Conference on Surface Plasmon Photonics, Copenhagen, 2019.

(27) Ummethala, S.; Pammi, V. A.; Uddin, A. H. M.; Hahn, L.; Freude, W.; Koos, C. Horizontal-Slot Plasmonic-Organic Hybrid (POH) Modulator. In Conference on Lasers and Electro-Optics; Optical Society of America, 2020.

(28) Ono, M.; Taniyama, H.; Xu, H.; Tsunekawa, M.; Kuramochi, E.; Nozaki, K.; Notomi, M. Deep-subwavelength plasmonic mode converter with large size reduction for Si-wire waveguide. Optica 2016, 3 (9), 999-999.

(29) Koch, U.; Messner, A.; Hoessbacher, C.; Heni, W.; Josten, A.; Baeuerle, B.; Ayata, M.; Fedoryshyn, Y.; Elder, D. L.; Dalton, L. R.; Leuthold, J. Ultra-Compact Terabit Plasmonic Modulator Array. J. Lightwave Technol. 2019, 37 (5), 1484-1491.

(30) Lee, H. W.; Papadakis, G.; Burgos, S. P.; Chander, K.; Kriesch, A.; Pala, R.; Peschel, U.; Atwater, H. a. Nanoscale Conducting Oxide PlasMOStor. Nano Lett. 2014, 14 (11), 6463-6468.

(31) Gramotnev, D. K.; Nielsen, M. G.; Tan, S. J.; Kurth, M. L.; Bozhevolnyi, S. I. Gap Surface Plasmon Waveguides with Enhanced Integration and Functionality. Nano Lett. 2012, 12 (1), 359-363.

(32) Robinson, B. H.; Johnson, L. E.; Elder, D. L.; Kocherzhenko, A. A.; Isborn, C. M.; Haffner, C.; Heni, W.; Hoessbacher, C.; Fedoryshyn, Y.; Salamin, Y.; Baeuerle, B.; Josten, A.; Ayata, M.; Koch, U.; Leuthold, J.; Dalton, L. R. Optimization of Plasmonic-Organic Hybrid ElectroOptics. J. Lightwave Technol. 2018, 36 (21), 5036-5047.

(33) Messner, A.; Eltes, F.; Ma, P.; Abel, S.; Baeuerle, B.; Josten, A.; Heni, W.; Caimi, D.; Fompeyrine, J.; Leuthold, J. Plasmonic Ferroelectric Modulators. J. Lightwave Technol. 2019, 37 (2), 281-290.

(34) Shimatsu, T.; Uomoto, M. Atomic diffusion bonding of wafers with thin nanocrystalline metal films. J. Vac. Sci. Technol., B: Nanotechnol. Microelectron.: Mater., Process., Meas., Phenom. 2010, 28 (4), 706-714.

(35) Pramassing, M.; Liebtrau, M.; Schill, H. J.; Irsen, S.; Linden, S. Interferometric near-field characterization of plasmonic slot waveguides in single- and poly-crystalline gold films. Opt. Express 2020, 28 (9), 12998-13007.

(36) Heni, W.; Haffner, C.; Elder, D. L.; Tillack, A. F.; Fedoryshyn, Y.; Cottier, R.; Salamin, Y.; Hoessbacher, C.; Koch, U.; Cheng, B.; Robinson, B.; Dalton, L. R.; Leuthold, J. Nonlinearities of organic 
electro-optic materials in nanoscale slots and implications for the optimum modulator design. Opt. Express 2017, 25 (3), 2627-2627.

(37) McPeak, K. M.; Jayanti, S. V.; Kress, S. J. P.; Meyer, S.; Iotti, S.; Rossinelli, A.; Norris, D. J. Plasmonic Films Can Easily Be Better: Rules and Recipes. ACS Photonics 2015, 2 (3), 326-333.

(38) Min, C.; Veronis, G. Theoretical investigation of fabricationrelated disorders on the properties of subwavelength metal-dielectricmetal plasmonic waveguides. Opt. Express 2010, 18 (20), 2093920948.

(39) Haffner, C.; Joerg, A.; Doderer, M.; Mayor, F.; Chelladurai, D.; Fedoryshyn, Y.; Roman, C. I.; Mazur, M.; Burla, M.; Lezec, H. J.; Aksyuk, V. A.; Leuthold, J. Nano-opto-electro-mechanical switches operated at CMOS-level voltages. Science 2019, 366 (6467), 860-864.

(40) Coudert, S.; Duchateau, G.; Dilhaire, S.; Lalanne, P. Attenuation of slow Metal-Insulator-Metal plasmonic waveguides, from Joule absorption to roughness-induced backscattering. arXiv 2018, arXiv:1810.07389; https://arxiv.org/abs/1810.07389 (accessed April 19, 2021).

(41) Ayata, M.; Fedoryshyn, Y.; Koch, U.; Leuthold, J. Compact, ultrabroadband plasmonic grating couplers. Opt. Express 2019, 27 (21), 29719-29729.

(42) Lumerical Inc. https://www.lumerical.com/products/ (accessed April 19, 2021).

(43) Marchetti, R.; Lacava, C.; Carroll, L.; Gradkowski, K.; Minzioni, P. Coupling strategies for silicon photonics integrated chips [Invited]. Photonics Res. 2019, 7 (2), 201-239.

(44) Raza, S.; Christensen, T.; Wubs, M.; Bozhevolnyi, S. I.; Mortensen, N. A. Nonlocal response in thin-film waveguides: Loss versus nonlocality and breaking of complementarity. Phys. Rev. B: Condens. Matter Mater. Phys. 2013, 88 (11), 115401.

(45) Porto, J. A.; García-Vidal, F. J.; Pendry, J. B. Transmission Resonances on Metallic Gratings with Very Narrow Slits. Phys. Rev. Lett. 1999, 83 (14), 2845-2848.

(46) García de Abajo, F. J. Colloquium: Light scattering by particle and hole arrays. Rev. Mod. Phys. 2007, 79 (4), 1267-1290.

(47) Garcia-Vidal, F. J.; Martin-Moreno, L.; Ebbesen, T. W.; Kuipers, L. Light passing through subwavelength apertures. Rev. Mod. Phys. 2010, 82 (1), 729-787.

(48) Ebbesen, T. W.; Lezec, H. J.; Ghaemi, H. F.; Thio, T.; Wolff, P. A. Extraordinary optical transmission through sub-wavelength hole arrays. Nature 1998, 391 (6668), 667-669.

(49) Lezec, H. J.; Degiron, A.; Devaux, E.; Linke, R. A.; MartinMoreno, L.; Garcia-Vidal, F. J.; Ebbesen, T. W. Beaming Light from a Subwavelength Aperture. Science 2002, 297 (5582), 820-822.

(50) Genet, C.; Ebbesen, T. W. Light in tiny holes. Nanoscience and Technology 2009, 205-212.

(51) Collin, S.; Pardo, F.; Teissier, R.; Pelouard, J. L. Horizontal and vertical surface resonances in transmission metallic gratings. Journal of Optics A: Pure and Applied Optics 2002, 4 (5), S154-S160.

(52) Collin, S.; Pardo, F.; Teissier, R.; Pelouard, J. L. Strong discontinuities in the complex photonic band structure of transmission metallic gratings. Phys. Rev. B: Condens. Matter Mater. Phys. 2001, 63 (3), 033107.

(53) Pang, Y.; Genet, C.; Ebbesen, T. W. Optical transmission through subwavelength slit apertures in metallic films. Opt. Commun. 2007, 280 (1), $10-15$.

(54) Marquier, F.; Greffet, J. J.; Collin, S.; Pardo, F.; Pelouard, J. L. Resonant transmission through a metallic film due to coupled modes. Opt. Express 2005, 13 (1), 70-76.

(55) Carretero-Palacios, S.; Mahboub, O.; Garcia-Vidal, F. J.; MartinMoreno, L.; Rodrigo, S. G.; Genet, C.; Ebbesen, T. W. Mechanisms for extraordinary optical transmission through bull's eye structures. Opt. Express 2011, 19 (11), 10429-42.

(56) Søndergaard, T.; Bozhevolnyi, S. I.; Novikov, S. M.; Beermann, J.; Devaux, E.; Ebbesen, T. W. Extraordinary Optical Transmission Enhanced by Nanofocusing. Nano Lett. 2010, 10 (8), 3123-3128.

(57) Messner, A.; Jud, P. A.; Winiger, J.; Heni, W.; Baeuerle, B.; Eppenberger, M.; Koch, U.; Haffner, C.; Xu, H.; Elder, D. L.; Dalton, L. R.; Ma, P.; Leuthold, J. High-Speed Plasmonic Modulator for
Simultaneous C- and O-Band Modulation with Simplified Fabrication. In Optical Fiber Communication Conference (OFC) 2020; Optical Society of America: San Diego, CA, 2020; p M1D.3.

(58) Heni, W.; Haffner, C.; Cottier, R.; Fedoryshyn, Y.; Elder, D. L.; Dalton, L. R.; Leuthold, J. Dielectric Layers in Plasmonic-Organic Hybrid Modulators. In Advanced Photonics 2018 (BGPP, IPR, NP, NOMA, Sensors, Networks, SPPCom, SOF), July 2, 2018, Zurich; Optical Society of America: Zurich, 2018; p IW3B.3.

(59) Xu, H.; Liu, F.; Elder, D. L.; Johnson, L. E.; de Coene, Y.; Clays, K.; Robinson, B. H.; Dalton, L. R. Ultrahigh Electro-Optic Coefficients, High Index of Refraction, and Long-Term Stability from Diels-Alder Cross-Linkable Binary Molecular Glasses. Chem. Mater. 2020, 32 (4), 1408-1421.

(60) Haffner, C.; Heni, W.; Elder, D. L.; Fedoryshyn, Y.; Đorđević, N.; Chelladurai, D.; Koch, U.; Portner, K.; Burla, M.; Robinson, B.; Dalton, L. R.; Leuthold, J. Harnessing nonlinearities near material absorption resonances for reducing losses in plasmonic modulators. Opt. Mater. Express 2017, 7 (7), 2168-2181. 